\title{
Optimierung der Schmerztherapie an neurologischen Stationen
}

\section{Optimising Pain Therapy for Neurological Inpatients}

Autoren

Institute
W. E. Wurm ${ }^{1,2}$, A. Lechner ${ }^{2}$, R. Schmidt ${ }^{2}$, I. S. Szilagyi ${ }^{3}$, C. Maier ${ }^{4}$, N. Nestler ${ }^{5}$, B. Pichler ${ }^{6}$, C. Foussek ${ }^{6}$, H. BornemannCimenti $^{3}$, A. Sandner-Kiesling ${ }^{3}$

Die Institutsangaben sind am Ende des Beitrags gelistet.

\section{Schlüsselwörter \\ - Neurologie \\ - Prävalenz \\ - Schmerzmessung \\ - Schmerzmanagement \\ - geplante \\ Behandlungsabläufe}

Key words

- neurology

- prevalence

- pain measurement

- pain management

- clinical pathways
Bibliografie

DOI http://dx.doi.org/

10.1055/s-0034-1399111

Fortschr Neurol Psychiatr 2015;

83: 149-156 @ Georg Thieme

Verlag KG Stuttgart . New York . ISSN 0720-4299

Korrespondenzadresse

Prof. Andreas SandnerKiesling

Universitätsklinik für Anästhesiologie und Intensivmedizin,

Medizinische Universität Graz

Auenbruggerplatz 29,

A-8036 Graz, Austria

andreas.sandner@

medunigraz.at

\section{Zusammenfassung \\ $\nabla$}

Hintergrund: An der Grazer Universitätsklinik für Neurologie wurde ein multiprofessionelles Schmerzmanagementkonzept eingeführt und anschließend mittels Befragungen von Patienten evaluiert.

Methoden: Therapieschemata wurden erstellt, die Schmerzmessung/-dokumentation wurde vereinheitlicht und die Mitarbeiter wurden geschult. Ergebnisse: Während des Aufenthalts traten bei 88,7\% der 63 befragten Patienten Schmerzen auf. Als Zeitpunkt besonders starker Schmerzen wurden am häufigsten die Nacht und der Vormittag gemeldet. Unter den besonders starke Schmerzen auslösenden Maßnahmen und Positionen wurde „Aufstehen/Gehen“ (19\%) am häufigsten genannt. Die höchsten Schmerzgrade traten bei Patienten mit degenerativen Erkrankungen der Wirbelsäule ohne Wurzelkompression auf.

Diskussion: Auf neurologischen Stationen stellen Schmerzen ein wichtiges Problem dar. Der Aufmerksamkeit bedürfen nächtliche Schmerzen, Schmerzen bei Mobilisation sowie die Schmerztherapie bei Patienten mit degenerativen Erkrankungen der Wirbelsäule.

\section{Hintergrund und Fragestellung \\ $\nabla$}

Schmerzen sind sowohl bei ambulant als auch bei stationär behandelten neurologischen Patienten ein sehr häufiges Problem [1, 2]. Das liegt zum einen daran, dass es sich bei vielen neurologischen Erkrankungen um primäre Schmerzerkrankungen handelt, zum anderen sind vor allem chronische Schmerzen häufig klinisch bedeutsame Komorbiditäten neurologischer Patienten und bedürfen eines biospsychosozialen Behandlungsansatzes [1-3]. Die Therapie von Schmerzen hat einen wachsenden Stellenwert im Aufgabenbereich von Fachärzten für Neurologie. Im deutschen Sprach-

\section{Abstract \\ $\nabla$}

Background: The Department of Neurology at the Medical University Graz has implemented a multiprofessional pain management concept and evaluated the outcome by means of a patient survey. Methods: Standard operating procedures for standardised pain measurement, documentation and therapy were developed. All engaged professional participants were trained before implementation.

Results: $88.7 \%$ of the surveyed 63 patients reported pain during the hospitalisation. During the night and in the morning, the occurrence of severe pain was most likely. The position or activity most likely triggering severe pain was mobilisation (19\%). Patients with degenerative diseases of the spine without radiculopathy reported the highest levels of pain.

Conclusions: Pain is an important problem for neurological inpatients. Nocturnal pain, pain induced by mobilisation, and pain therapy for patients with degenerative diseases of the spine without radiculopathy require particular attention.

raum kam es in den letzten Jahren zu einer zunehmenden Verbesserung des schmerztherapeutischen Angebots an neurologischen Kliniken. So konnte 2007 in einer Umfrage zur Strukturqualität der Schmerztherapie an 391 neurologischen Abteilungen in Deutschland erhoben werden, dass nahezu alle Kliniken Schmerzpatienten speziell zur Diagnostik und Therapie aufnehmen, dass fast 90\% der Neurologen eine ausführliche Schmerzanamnese erheben und dass etwa zwei Drittel der Kliniken sowohl multidimensionale als auch psychometrische Schmerzfragebögen verwenden [3]. Die Autoren empfehlen eine weitere Optimierung der Abläufe für stationär aufgenommene neurolo- 
gische Patienten und kritisieren die dürftige Datenlage bezüglich der Ergebnisqualität optimierter Schmerzmanagementabläufe an neurologischen Kliniken [3].

Im Rahmen des Projekts „Schmerzfreies Krankenhaus“ wurden an 25 Kliniken in Deutschland umfassende Maßnahmen zur Verbesserung des Schmerzmanagements durchgeführt [4]. Infolge spezieller Gegebenheiten bei stationär behandelten neurologischen Patienten wie hohes Lebensalter und Einschränkungen von Mobilität oder Kommunikationsfähigkeit [1,5 - 8] stellten wir die Hypothese auf, dass es an neurologischen Abteilungen erforderlich ist, diesen speziellen Problemen der Patienten gerecht zu werden, um ihnen eine optimale Schmerztherapie ermöglichen zu können. Das Ziel unserer Untersuchung bestand darin, infolge der Implementierung eines multiprofessionellen Schmerzmanagementkonzepts an der neurologischen Universitätsklinik der Medizinischen Universität Graz die Ergebnisqualität der erfolgten Maßnahmen zu evaluieren, um einen Optimierungsbedarf in der Schmerztherapie an neurologischen Kliniken zu erheben.

\section{Studiendesign und Untersuchungsmethoden \\ $\nabla$}

\section{Studiendesign}

Eine prospektive Fragebogenerhebung von Patienten erfasste 2010 die erforderlichen schmerzbezogenen Informationen für die Analyse eines Optimierungsprozesses des Schmerzmanagements der Universitätsklinik für Neurologie der Medizinischen Universität Graz.

\section{Setting}

Eine multiprofessionelle Arbeitsgruppe, bestehend aus Ärzten, Psychologen, Pflegenden, Physiotherapeuten und Ergotherapeuten entwickelte seit 2008 am LKH-Univ.-Klinikum Graz abgestufte Therapieschemata für die medikamentöse Schmerztherapie und nichtmedikamentöse Maßnahmen sowie die Therapie und Prophylaxe von Nebenwirkungen. Inhaltlich orientierten sich diese Standards in erster Linie an aktuellen Leitlinien und Algorithmen von Fachgesellschaften sowie an klinikinternen Gegebenheiten wie z. B. Verfügbarkeit von Medikamenten und nichtmedikamentösen Verfahren. Bei den Therapieschemata wurde besonderer Wert auf Übersichtlichkeit und leichte Umsetzbarkeit gelegt. Die Therapieschemata sind im vollen Umfang unter http://dx.doi.org/10.1055/s-0034-1399111 enthalten.

Schmerzerfassung, -dokumentation, -anamnese und -therapie wurden klinikweit vereinheitlicht und intensiv geschult. Für die Schmerzerfassung bei der Aufnahme wurde ein Fragebogen erarbeitet und für die Schmerzmessung ein eigenes Lineal entwickelt (modifizierte numerische Ratingskala; NRS). Die mindestens 3-mal täglich erhobenen Schmerzwerte wurden auf der Fieberkurve notiert: Ruheschmerz und Maximalschmerz in den letzten 24 Stunden oder Belastungsschmerz. Die Interventionsgrenzen wurden für den Ruheschmerz mit NRS 3, für den Maximalschmerz mit NRS 4 und für den Belastungsschmerz mit NRS 5 definiert. Durch die Verankerung der Interventionsgrenzen in unseren Therapieschemata konnten dem zuständigen Personal einheitlich und klar nachvollziehbare Interventionsstrategien an die Hand gegeben werden. Sämtliche Vorlagen wurden in allen Stationsstützpunkten und Behandlungsräumen hinterlegt und allen Beteiligten in elektronischer Form zur Verfügung gestellt. Nach Schulung, Implementierung und Anwendung des gesamten Konzepts erfolgte die externe Evaluierung im Rahmen der Zertifizierung „Certkom-Qualifizierte Schmerztherapie“ mittels Pa- tientenbefragung, medizinischer Kennbögen, interner und externer Audits, Stationsbegehungen, Stichproben in Fieberkurven und Krankengeschichten. Die Patientenbefragung erfolgte dabei in Papierform, unterstützt durch Mitarbeiter der klinikinternen Stabsstelle „Qualitätsmanagement/Risikomanagement“ mit im Projekt „Schmerzfreies Krankenhaus“ validierten, standardisierten Fragebögen [4]. Zusätzlich wurden für alle teilnehmenden Patienten mittels medizinischer Kennbögen demografische Daten, Diagnosen, aktuelle Analgetikatherapie und Nebenwirkungen erfasst. Zur Auswahl der Patienten standen alle während des Erhebungszeitraums (01.08.2010 - 30.09.2010) auf den 3 allgemeinen neurologischen Stationen der Grazer Universitätsklinik für Neurologie behandelten Patienten. Die Einschlusskriterien, Ausschlusskriterien sowie Primär- und Sekundärvariablen der Studie sind in 0 Tab. 1 dargestellt. Die Befragung erfolgte stichpunktprävalenzartig an 7 unterschiedlichen Arbeitstagen.

Auf den 3 beteiligten Stationen bestand ein hoher Durchsatz, wobei die Subgruppe der Schlaganfallpatienten längere Verweildauern aufwies als Patienten der anderen Diagnosegruppen. Auf der Stroke Unit und Intensivstation wurden keine Patientenbefragungen durchgeführt. Manche der befragten Patienten auf der Normalstation wurden jedoch vorher auf der Stroke Unit oder Intensivstation behandelt. Vermutlich lassen Patientenantworten teilweise auch auf den Behandlungszeitraum vor der Transferierung auf die Normalstation rückschließen. Eine Randomisierung wurde nicht durchgeführt, um ein möglichst breites Bild der befragten Patienten zu bekommen.

\section{Statistik}

Die statistische Auswertung erfolgte anonymisiert durch die CERTKOM e. V. unter Verwendung von T-Tests, Chi-Quadrat-Tests

Tab. 1 Einschluss-bzw. Ausschlusskriterien, Primär- und Sekundärvariablen der im Rahmen der Studie befragten neurologischen Patienten.

\begin{tabular}{|c|c|}
\hline Einschlusskriterien & $\begin{array}{l}\text { Eingeschlossen wurden alle an } 7 \text { Stichtagen im } \\
\text { Zeitraum von } 8 \text { Wochen im Sommer/Herbst } 2010 \\
\text { an } 2 \text { Stationen der Universitätsklinik für Neurolo- } \\
\text { gie der medizinischen Universität Graz betreuten } \\
\text { Patienten, die der deutschen Sprache mächtig und } \\
\text { fähig zur eigenständigen Beantwortung der Fra- } \\
\text { gen waren, unabhängig davon, ob sie zu diesem } \\
\text { Zeitpunkt Schmerzen hatten oder nicht. }\end{array}$ \\
\hline \multirow[t]{6}{*}{ Ausschlusskriterien } & Fehlende Zustimmung \\
\hline & Zu starke Beeinträchtigung des Allgemeinzustands \\
\hline & Eingeschränktes Denkvermögen \\
\hline & Alter unter 18 Jahre \\
\hline & Psychiatrische Erkrankung \\
\hline & Abwesenheit \\
\hline \multirow[t]{3}{*}{ Primärvariablen } & Häufigkeit und Intensität von Schmerzen \\
\hline & Erkrankungen mit den höchsten Schmerzwerten \\
\hline & $\begin{array}{l}\text { Tageszeitliche Bindung und auslösende Maßnah- } \\
\text { men/Positionen besonders starker Schmerzen }\end{array}$ \\
\hline \multirow[t]{7}{*}{ Sekundärvariablen } & Prozessqualität: \\
\hline & Wurden bei Schmerzen Medikamente gegeben? \\
\hline & $\begin{array}{l}\text { Wurden bei Meldung wegen starker Schmerzen } \\
\text { zusätzliche, ausreichend wirksame Medikamente } \\
\text { gegeben? }\end{array}$ \\
\hline & Wie war die Reaktionszeit bei Meldung des \\
\hline & Patienten? \\
\hline & $\begin{array}{l}\text { Bewertung der Schmerztherapie durch die } \\
\text { Patienten }\end{array}$ \\
\hline & $\begin{array}{l}\text { Informiertheit, Aktivität und Meldeverhalten der } \\
\text { Patienten }\end{array}$ \\
\hline
\end{tabular}


und deskriptiv-explorativen Berechnungen. Zusätzliche Datenanalysen erfolgten mittels Whitney-U-Testungen und explorativen Datenanalysen an der Medizinischen Universität Graz. Die Ergebnisse der Patienten- und Mitarbeiterbefragung wurden dabei mit Daten anderer, bereits von der CERTKOM e.V. zertifizierter Kliniken verglichen. Bei den Vergleichskliniken handelte es sich um konservative Abteilungen in erster Linie des Fachbereichs Innere Medizin und zu einem geringeren Anteil um neurologische Kliniken. Von den insgesamt 1100 Referenzpatienten dieser Kliniken waren 311 (28,3\%) neurologische Patienten. Die Darstellung erfolgte in Prozent aller befragten Patienten bzw. als Median \pm Perzentile inkl. Ausreißer.

\section{Ergebnisse}

$\nabla$

\section{Epidemiologische Daten der befragten Patienten}

Von den 85 ausgeteilten Fragebögen wurden 63 ausgefüllt retourniert. Von den Befragten waren 63,3\% männlichen, 36,7\% weiblichen Geschlechts. Die am häufigsten vertretene Altersklasse waren die 40 - bis 70 -Jährigen mit 50,8\%. Am zweithäufigsten waren die über 70 -jährigen Patienten mit 36,5\% vertreten. Die Zuordnung der Patienten zu ICD-10-Diagnosen ist in O Tab. 2 dargestellt.

\section{Häufigkeit und Intensität von Schmerzen}

Nur 23 Patienten (38,3\%) waren vor der Aufnahme schmerzfrei. Von den befragten Patienten bestätigten 30,6\%, vor dem Krankenhausaufenthalt regelmäßig schmerzstillende Medikamente eingenommen zu haben. Während des Aufenthalts traten bei 88,7\% der befragten Patienten Schmerzen auf. Jeder zweite Patient gab an, vor der Aufnahme schon Analgetika eingenommen zu haben ( $\bullet$ Tab. 3). Intrahospital sieht man generell eine Zunahme der verordneten Analgetika, speziell aber der Co-Analgetika. Die am häufigsten beschriebenen Schmerzen waren: Rücken(48,3\%), Kopf- (45,8\%) und Nackenschmerzen (38,6\%). Bei der Subgruppe der Schlaganfallpatienten traten Schmerzen ebenfalls

Tab. 2 Zuordnung der befragten Patienten zu ICD-10-Diagnosen. Alle Patienten wurden nur jeweils einer Schmerzdiagnose zugeordnet, basierend auf den erhobenen Haupt- bzw. Nebendiagnosen.

\begin{tabular}{|c|c|}
\hline Diagnose & Häufigkeit (n) \\
\hline Ischämischer Insult 163. & 16 \\
\hline $\begin{array}{l}\text { Sonstige Krankheiten der Wirbelsäu- } \\
\text { le und des Rückens (ohne Myelon- } \\
\text { oder Wurzelkompression) M53. }\end{array}$ & 9 \\
\hline Epilepsie G40. & 7 \\
\hline Hirntumoren C71. + D33. & 5 \\
\hline Neuroborreliose A69. & 4 \\
\hline Sonstige Meningitiden G03. & 4 \\
\hline $\begin{array}{l}\text { Degenerative Krankheiten der Wir- } \\
\text { belsäule mit Wurzelkompression } \\
\text { M50.1 + M.51.1 }\end{array}$ & 3 \\
\hline \multicolumn{2}{|c|}{ Seltener $(n \leq 2)$ traten folgende Erkrankungen auf: } \\
\hline Arthrose M19. & Alkoholkrankheit F10. \\
\hline $\begin{array}{l}\text { Erkrankungen der Augen und Augen- } \\
\text { anhangsgebilde H.57 }\end{array}$ & Guillain-Barré-Syndrom G61. \\
\hline Spannungskopfschmerz G44.2 & Sonstige Kopfschmerzen G44. \\
\hline Multiple Sklerose G35. & Osteoporose M81. \\
\hline Parkinson-Syndrom G20.+G21. & St.p. Poliomyelitis B91 \\
\hline
\end{tabular}

am häufigsten in diesen Lokalisationen auf. Deutlich weniger Patienten unserer Klinik überschritten die im Projekt „Schmerzfreies Krankenhaus“ bestätigten Interventionswerte für Ruhe, Belastungs- und Maximalschmerzen als Patienten in den zertifizierten Vergleichsklinken. Ein zu hoher Ruheschmerz wurde von 15,0\% der befragten Patienten (in den Vergleichskliniken von 36,4\%), ein zu hoher Belastungsschmerz von 16,9\% der befragten Patienten (in den Vergleichskliniken von 40,5\%) und ein zu hoher Maximalschmerz in den letzten 24 Stunden von $26,7 \%$ der befragten Patienten (in den Vergleichskliniken von 43,7\%) angegeben ( $\bullet$ Abb. 1). Einen zu hohen Ruheschmerz meldeten 9,7\%, einen zu hohen Belastungsschmerz 19,4\% und einen zu hohen Maximalschmerz 25,8\% der Schlaganfallpatienten.

Tab.3 Anzahl der Patienten ( $n=63)$, die vor oder während der stationären Behandlung Analgetika und Coanalgetika eingenommen haben. Die Zahlen entsprechen jeweils der Anzahl der Patienten, die während des angegebenen Zeitraums Medikamente der genannten Substanzgruppe (n) eingenommen haben.

\begin{tabular}{|c|c|c|}
\hline Substanzgruppen & $\begin{array}{l}\text { Vor dem } \\
\text { Aufenthalt } \\
\text { (n) }\end{array}$ & $\begin{array}{l}\text { Während des } \\
\text { Aufenthalts } \\
\text { (n) }\end{array}$ \\
\hline Nicht saure Nichtopioidanalgetika & 4 & 17 \\
\hline Nichtsteroidale Antirheumatika & 13 & 21 \\
\hline Opiate & 10 & 18 \\
\hline Trizyklische Antidepressiva & 1 & 5 \\
\hline $\begin{array}{l}\text { Sonstige analgetisch wirksame } \\
\text { Antidepressiva }\end{array}$ & 2 & 7 \\
\hline Antiepileptika & 9 & 13 \\
\hline Muskelrelaxantien & 3 & 12 \\
\hline Triptane & 2 & 2 \\
\hline Glukocorticoide & 2 & 8 \\
\hline Analgetika und Coanalgetika (total) & 31 & 40 \\
\hline
\end{tabular}

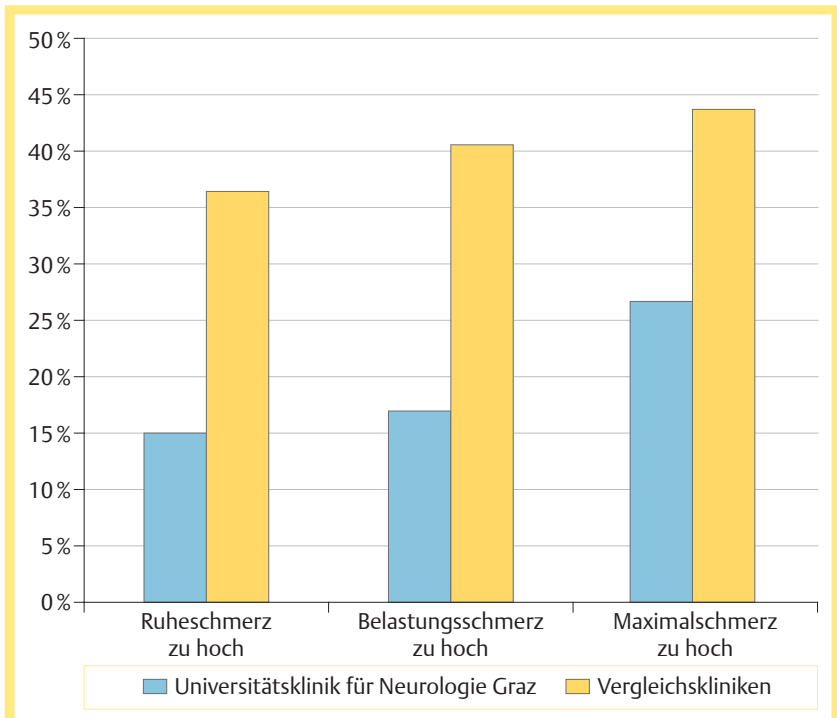

Abb. 1 Anteil der Patienten (in \%) mit zu hohen Schmerzangaben, bezogen auf die im Projekt „Schmerzfreies Krankenhaus“ erhobenen Interventionswerte für Ruheschmerz (NRS 3), Belastungsschmerz (NRS 5) und Maximalschmerz (NRS 4). Bei den Vergleichskliniken handelte es sich um von CERTKOM e. V. bereits zertifizierte internistische und neurologische Abteilungen. Von den insgesamt 1100 Referenzpatienten dieser Kliniken waren $311(28,3 \%)$ neurologische Patienten. 


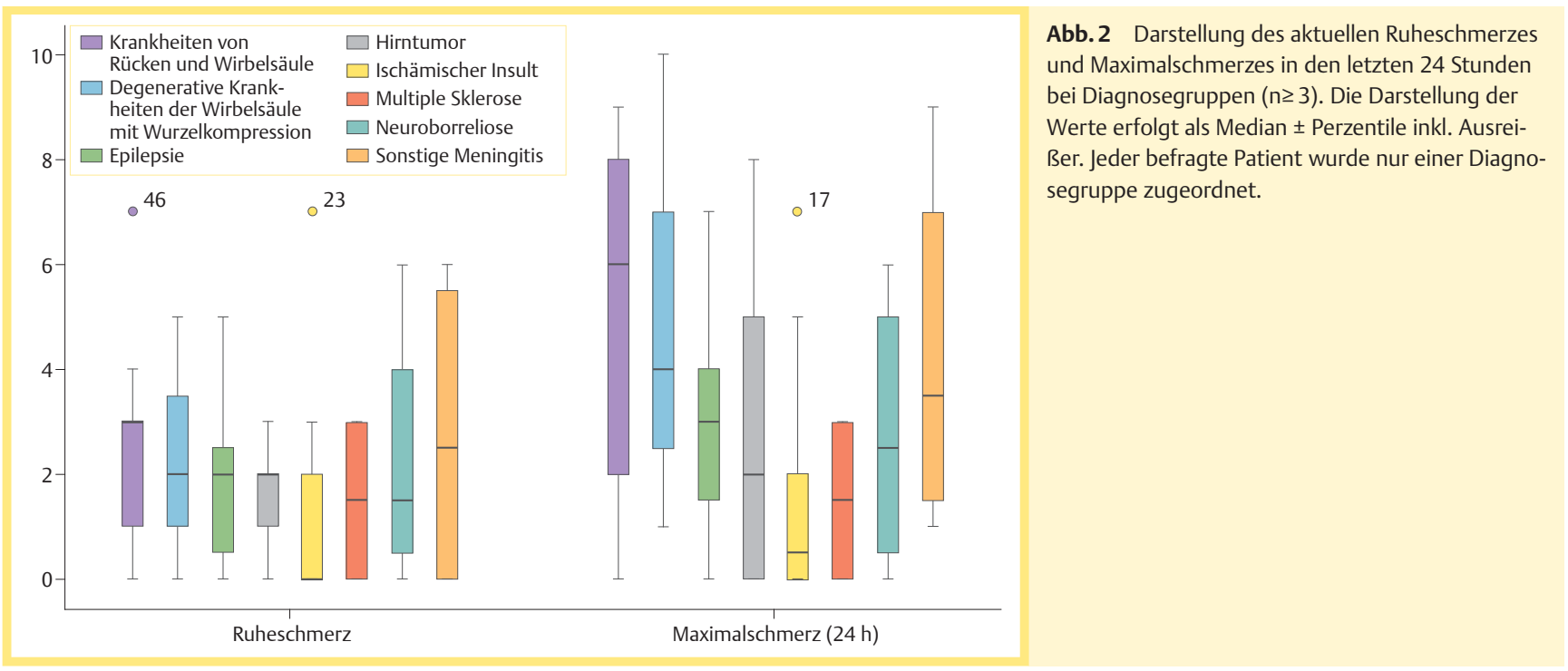

Erkrankungen mit den höchsten Schmerzwerten

Die höchsten Ruheschmerz- und Maximalschmerz-Durchschnittswerte in den letzten 24 Stunden nannten Patienten mit sonstigen (ohne Myelon-/Wurzelkompression) Krankheiten der Wirbelsäule und des Rückens. Die zweithöchsten Ruheschmerz-Werte gaben Patienten mit sonstiger Meningitis an. Für den Maximalschmerz konnten bei Patienten mit degenerativen Krankheiten der Wirbelsäule mit Wurzelkompression die zweithöchsten Durchschnittswerte erhoben werden, gefolgt von Patienten mit sonstiger Meningitis. Die niedrigsten Werte für Ruheschmerz und Maximalschmerz gaben Patienten mit ischämischem Insult an. Den zweitniedrigsten Ruheschmerz gaben Patienten mit multipler Sklerose und Patienten mit Neuroborreliose an ( $\bullet$ Abb. 2).

\section{Tageszeitliche Bindung und auslösende Maßnahmen/ Positionen besonders starker Schmerzen}

Als Zeitpunkt besonders starker Schmerzen wurden am häufigsten die Nacht und der Vormittag mit je $23,3 \%$ gemeldet. Nur $10 \%$ der Patienten berichteten über eine besonders hohe Schmerzintensität am Nachmittag und 43,3\% gaben keine tageszeitlichen Schwankungen an ( $\bullet$ Abb. 3). Als besonders starke Schmerzen auslösende Anlässe im Sinne von Behandlungen oder von Körperpositionen und Bewegungen wurden „Aufstehen/Gehen“ (19\%) sowie „im Liegen“ (11,1\%) am häufigsten genannt ( $\bullet$ Abb. 3 ).

\section{Prozessqualität}

Bei Schmerzen erhielten $60 \%$ der Patienten eine regelmäßige Gabe von Analgetika und 57,9\% eine zusätzliche Bedarfsgabe bei fester Anordnung. Bei besonders starken Schmerzen erhielten $70,6 \%$ der Patienten zusätzliche, ausreichend wirksame schmerzstillende Medikamente. Innerhalb von 30 Minuten nach Schmerzmeldung erhielten tagsüber 59,4\%, und in der Nacht $50 \%$ der Patienten ihre Medikamente. Als zu kurz wirksam wurden die Schmerzmedikamente von 20,0\% der befragten Patienten bezeichnet (in den Vergleichskliniken von nur 12,9\%). Fünfundzwanzig Prozent der befragten Patienten erhielten laut ihren Aussagen vor schmerzauslösenden Maßnahmen zusätzliche schmerzstillende Medikamente.
Bewertung der Schmerztherapie durch die Patienten Als wirksam beurteilten 68,6\% der behandelten Patienten ihre Schmerztherapie, das ist ein etwas besserer Wert, als in den zertifizierten Vergleichskliniken erhoben werden konnte (66,8\%). Im Durchschnitt wurde die Schmerztherapie auf einer Schulnotenskala (1 -6) mit "gut" bewertet. Von den befragten Patienten gaben $62,7 \%$ an, keine Nebenwirkungen durch die Schmerzmedikamente bemerkt zu haben.

\section{Informiertheit, Aktivität und Meldeverhalten der Patienten}

Von den befragten Patienten gaben 55,2\% an, sehr gut über ihre Schmerztherapie informiert worden zu sein. Auf der anderen Seite gaben $50,9 \%$ an, nicht über eigene Aktivitäten zur Schmerzlinderung aufgeklärt worden zu sein. Beim Auftreten von Schmerzen meldeten sich 52,9\% der befragten Patienten beim Pflegepersonal. Erst bei stärkeren Schmerzen meldeten sich 26,5\%. Nur wenige Patienten meldeten sich trotz aufgetretener Schmerzen gar nicht (o Abb. 4).

\section{Diskussion \\ $\nabla$}

Das ist der erste Bericht über die Häufigkeit von Schmerzen, schmerzauslösende Faktoren und tageszeitliche Bindungen von starken Schmerzen bei den unterschiedlichen an einer neurologischen Universitätsklinik stationär behandelten Patientengruppen.

\section{Häufigkeit und Intensität von Schmerzen}

Der Prozentsatz unserer Patienten, die während des Aufenthalts unter Schmerzen litten (88,7\%), liegt im obersten Bereich der Vergleichsliteratur. Diese beschreibt dafür je nach Fachrichtung eine Inzidenz von $40-90 \%[1,2,9,10]$. Die Erklärung für diese Ergebnisse mag teilweise im deutlich erhöhten Durchschnittsalter (60 Jahre) unseres Patientenkollektivs im Vergleich zum Bevölkerungsdurchschnitt liegen. Neurologische Patienten leiden aber auch abgesehen von altersbezogenen Faktoren deutlich häufiger an Schmerzen als die Allgemeinbevölkerung. So gaben etwa in einer Befragung in Schottland zwei Drittel von ambulant versorgten neurologischen Patienten an, in den 2 Wochen unmittelbar vor dem Befragungszeitpunkt unter Schmerzen gelitten zu 


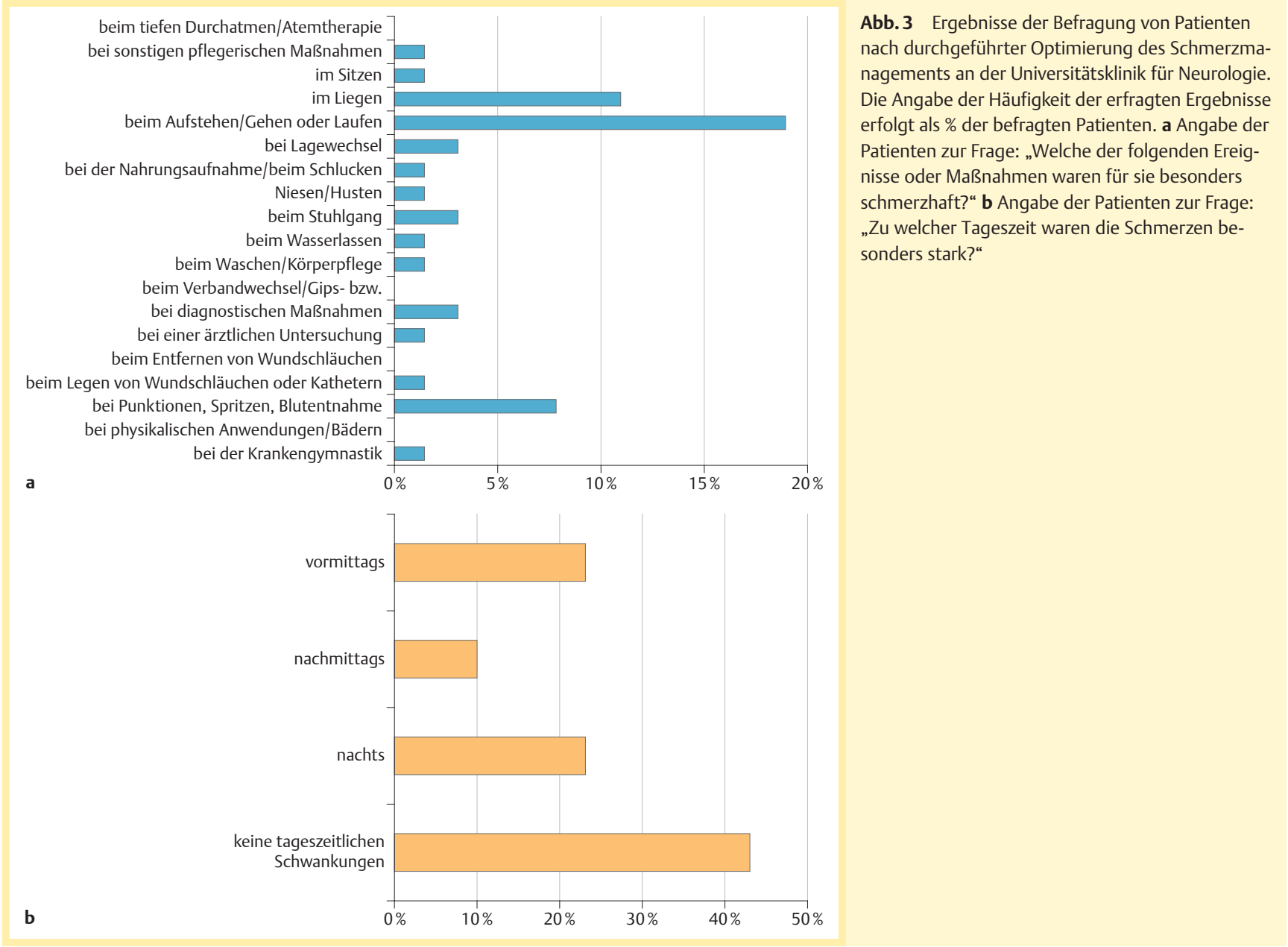

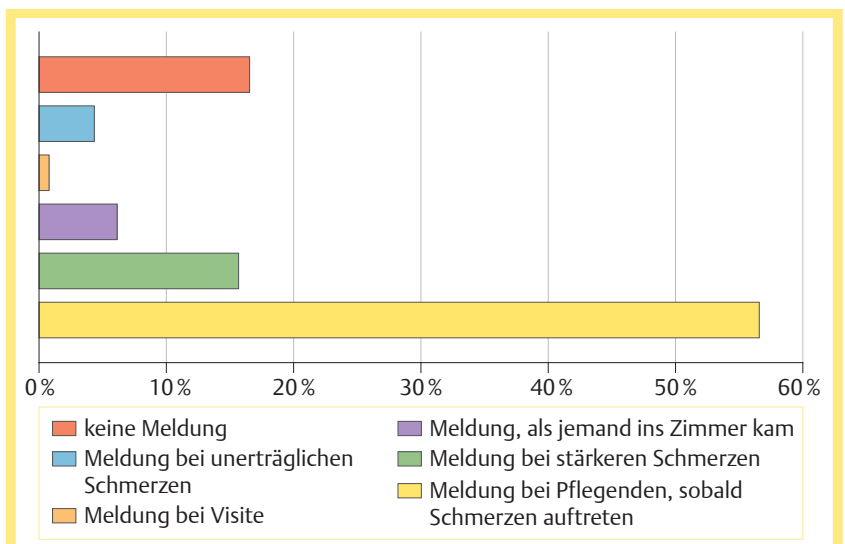

Abb. 4 Meldeverhalten der Patienten beim Auftreten von Schmerzen. Die Angabe der Häufigkeit der erfragten Ergebnisse erfolgt als \% der befragten Patienten.

haben [2], und in einer Befragung von 400 stationär behandelten neurologischen Patienten an einer deutschen Universitätsklinik konnte erhoben werden, dass $80 \%$ der Patienten vor der Aufnahme unter Schmerzen gelitten hatten [1]. Da viele neurologische Erkrankungen, wie etwa Polyneuropathien, Nerven-/ Wurzelkompressionssyndrome, Kopfschmerzen und Hirnhautentzündungen durch Schmerzen charakterisiert sind, ist es nicht verwunderlich, dass die meisten neurologischen Kliniken Patienten zur Diagnostik und Therapie von Schmerzen aufnehmen [3]. Die in Graz beobachteten häufigsten Schmerzlokalisationen (Rücken vor Kopf und Nacken) entsprachen den Ergebnissen von Gerbershagen et al. [1]. Wenn auch unsere Klinik diesbezüglich ein deutlich besseres Ergebnis als die bereits von der CERTKOM e.V. zertifizierten Vergleichskliniken erzielen konnte ( $\bullet$ Abb.1), die hohe Inzidenz an therapiebedürftigen Maximalschmerzen gilt es in Zukunft zu reduzieren.

\section{Erkrankungen mit den höchsten Schmerzwerten} Interessanterweise wurden die stärksten Ruheschmerzen und Maximalschmerzen von Patienten mit Rückenschmerzen ohne Myelon- oder Wurzelkompression genannt. Auch Patienten mit Rückenschmerzen infolge von Wurzelkompressionen gaben geringere Ruhe- und Maximalschmerzen an. Wir können nur vermuten, dass die relativ hohe Schmerzintensität dieser Patienten als Ausdruck der bio-psycho-sozialen Bedingtheit dieser Beschwerden interpretiert werden kann. Eine weitere Ursache mag darin liegen, dass nichtspezifische Rückenschmerzen bei den verschiedenen medizinischen Professionen weniger Beachtung finden als Schmerzen anderer Ätiologie und folglich häufiger inadäquat therapiert werden. Ein ähnliches Phänomen zeigt sich in der besseren schmerztherapeutischen Versorgung von Patienten mit Tumorschmerzen im Vergleich zu Patienten, die an Schmerzen anderer Ätiologie leiden [4]. Das Auftreten besonders starker Ruheschmerzen bei den Patienten mit Meningi- 
tis lässt sich gut mit der aus der Literatur bekannten hohen Prävalenz von starken Kopf- und Nackenschmerzen bei Patienten mit Hirnhautentzündungen unterschiedlicher Ätiologie erklären $[11,12]$. Dass bei diesen Patienten der durchschnittliche Maximalschmerz nicht deutlich über dem durchschnittlichen Ruheschmerz lag, mag an der eingeschränkten Mobilität dieser Patienten gelegen haben.

\section{Tageszeitliche Bindung besonders starker Schmerzen}

Erklärungen für die häufige Nennung der Nacht als Zeitpunkt besonders starker Schmerzen können in der Anwendung von Analgetika mit zu kurzer Halbwertszeit, einer zu frühen Gabe (z.B. zum Abendessen um 16.30 Uhr) oder zu geringer Dosierung der Analgetika am Abend mit nächtlichem Spiegelabfall liegen [13, 14]. Ebenso können zu geringes oder verzögertes Meldeverhalten der Patienten, eine fatalistische Einstellung der Patienten, fehlende Behandlungsalgorithmen oder zu lange Reaktionszeiten beim Personal infolge eingeschränkter Personalressourcen im Nachtdienst eine Rolle spielen [15]. Seitens der Patienten könnte eine Einschränkung der Mobilität und damit der Befähigung zum selbstständigen Lagewechsel, ein häufiges Problem von stationär behandelten neurologischen Patienten, eine Erklärung für das häufige Auftreten nächtlicher Schmerzspitzen sein [7, 8]. Eine weitere Ursache für gehäuftes Auftreten besonders starker Schmerzen in der Nacht kann in einer depressiven Komorbidität von Patienten liegen. Auch bei nichtdepressiven Patienten sind Schlafstörungen ausgesprochen problematisch, da sie sich negativ auf affektiv-motivationale, aber auch kognitive Prozesse auswirken [16 - 18], was sich in der Folge negativ auf den Rehabilitationsprozess neurologischer Patienten auswirken könnte.

\section{Was löst besonders starke Schmerzen aus?}

Dass Schmerzen besonders häufig beim Aufstehen und Gehen auftraten, ist naheliegend. So treten postpunktionelle Kopfschmerzen vor allem beim Aufstehen und Gehen auf [19, 20]. Der Einfluss der Mobilisation sowohl auf Patienten mit radikulären und nichtspezifischen Rückenschmerzen als auch auf Patienten mit Paresen muss differenzierter betrachtet werden. Häufig tritt bei diesen Patienten während der Mobilisation eine akute Schmerzzunahme auf. Die Zunahme von radikulären und nichtradikulären Rückenschmerzen bei Mobilisation ist durch eine passagere Zunahme der mechanischen Kompression bzw. mechanischen Überlastung, Muskelkontraktion, Entzündungsreaktion etc. leicht erklärbar. Andererseits spielt die Mobilisation eine zentrale Rolle in der Prophylaxe und Therapie von Muskelatrophie und daraus resultierenden funktionellen Defiziten und chronischen Schmerzen [6, 21 - 24]. In diesem Zusammenhang ist eine prophylaktische Gabe von Analgetika vor Mobilisationsmaßnahmen sinnvoll, wenn Patienten hohe Schmerzgrade für den Belastungsschmerz angeben. Interessanterweise wurde als zweithäufigste schmerzauslösende Position oder Maßnahme „im Liegen“ genannt. Einerseits dürften dabei Faktoren, die auch für das häufige Auftreten nächtlicher Schmerzspitzen verantwortlich sind, eine zentrale Rolle spielen. Anderseits kann man annehmen, dass auch tagsüber eine optimale physiotherapeutische und pflegerische Betreuung mittels entsprechender Lagerungs- und Mobilisationsmaßnahmen bei neurologischen Patienten einen wesentlichen schmerzpräventiven Effekt hat $[21-24]$.

\section{Prozessqualität}

Problematisch ist die häufige Meldung von besonders starken Schmerzen in der Nacht. Ein wichtiges Qualitätskriterium für eine optimierte Schmerztherapie ist, dass Analgetika mit ausreichend langer Halbwertszeit nach festem Zeitplan (Dosierungsintervall) gegeben werden, damit es im Verlauf des Tages und vor allem der Nacht zu keinem Abfall des Serumspiegels mit der Konsequenz der Schmerzzunahme kommt $[13,14]$. Die sogenannte bedarfsorientierte Gabe sollte nur bei sehr wenigen Patienten mit sehr seltenen Schmerzen angeordnet werden. Entsprechend den oben genannten Ergebnissen der Patientenbefragung besteht diesbezüglich an unserer Klinik ein weiterer Optimierungsbedarf.

Bedauerlicherweise mussten wir feststellen, dass unsere Patienten mit der Anforderung von Bedarfsmedikation mehr überfordert als unterstützt waren. Wir haben daher in der Folge bei mehreren Schmerzmeldungen die Basismedikation erhöht.

\section{Bewertung der Schmerztherapie durch die Patienten}

Dass etwa zwei Drittel der befragten Patienten ihre Schmerztherapie als wirksam bezeichneten und dass die Schmerztherapie im Durchschnitt auf einer Schulnotenskala (1 - 6) mit „gut“ bewertet wird, ist im Vergleich mit den Ergebnissen der konservativen Vergleichskliniken ein akzeptables Ergebnis. Bekanntlich treten bei älteren Menschen Analgetikanebenwirkungen und -interaktionen besonders häufig auf [25 - 27]. In diesem Zusammenhang ist positiv zu vermerken, dass fast zwei Drittel der Patienten keine Nebenwirkungen infolge der Schmerztherapie angaben.

\section{Informiertheit, Aktivität und Meldeverhalten der Patienten}

Optimierungsbedarf besteht in der Aufklärung und Information von Patienten über Möglichkeiten der Schmerzlinderung und speziell vor allem über Aktivitäten, die sie selbstständig durchführen können, da das Gefühl von Selbstwirksamkeit einen präventiven Effekt gegen die Chronifizierung von Schmerzen hat [28]. Ein großer Anteil stationär behandelter neurologischer Patienten befindet sich in fortgeschrittenem Lebensalter. Gerade Menschen dieser Altersgruppe haben oft Hemmungen, ihre Schmerzen adäquat zu verbalisieren [15]. Um bei stärkeren Schmerzen von Seiten des medizinischen Personals aktiv eingreifen zu können, bedarf es einer engen Zusammenarbeit zwischen Patienten und Mitarbeitern einer Klinik [29]. Deshalb war ein zentrales Ziel unserer Interventionen zur Optimierung des Schmerzmanagements, die Kommunikation mit den Patienten zu verbessern.

\section{Limitationen}

Limitiert wird die Aussagekraft unserer Studie durch die geringe Fallzahl sowie durch die Tatsache, dass es sich um eine Querschnittsstudie mit nur 7 Erhebungszeitpunkten handelt und für den Zeitpunkt vor der Zertifizierung keine Ausgangswerte vorliegen. Leider war nur ein Vergleich unserer Daten mit Patienten aller konservativen Vergleichskliniken möglich. Von den insgesamt 1100 Referenzpatienten dieser Kliniken waren 311 (28,3\%) neurologische Patienten, die Datensätze waren jedoch nicht mehr von den internistischen trennbar. Die Aussagekraft des Vergleichs mit den internistischen Patienten ist eingeschränkt, da bekanntlich wesentliche Unterschiede im Auftreten verschiedener Schmerzarten zwischen internistischen und neurologischen Patienten bestehen. Andererseits werden auch auf internen Stationen neurologi- 
sche Patienten behandelt, wenn im befragten Krankenhaus keine eigene neurologische Station oder Abteilung vorhanden ist.

Unsere erhobenen Daten sind in ihrer Zusammensetzung nicht direkt mit anderen neurologischen Kliniken vergleichbar, da die Zusammensetzung der Patientenpopulationen sich abhängig vom fachlichen Schwerpunkt unterscheiden kann.

Es wurde nicht zwischen akuten und chronischen Schmerzen unterschieden sowie keine gezielte Erhebung von Symptomen der Angst und Depression mittels validierter Tests durchgeführt. Psychiatrische Vorerkrankungen stellten ein Ausschlusskriterium für die Studie dar.

Limitiert werden unsere Ergebnisse auch durch den Geschlechterbias (63,3\% männlich und $37 \%$ weiblich), da es geschlechtsspezifische Unterschiede sowohl in der Häufigkeit und Verarbeitung von Schmerzen als auch in der Wirksamkeit und Verträglichkeit von analgetischen Therapien und nichtmedikamentösen Maßnahmen gibt.

Außerdem müssen potenzielle Verzerrungen infolge von Selektionsbias, unvollständigem Antwortverhalten oder tendenziellem Antwortverhalten bei einer retrospektiven Befragung aufgrund von bereits wirksam gewordenen Bewältigungsstrategien zur besseren Verarbeitung des Krankenhausaufenthalts beachtet werden [30].

Aus unserer Studie geht hervor, dass Schmerzen ein sehr häufiges und klinisch immens relevantes Problem bei stationär behandelten neurologischen Patienten sind. Von essenzieller Bedeutung für eine erfolgreiche Schmerztherapie an stationären neurologischen Abteilungen ist ein interdisziplinärer Therapieansatz, in dessen Zentrum die Kommunikation mit dem Patienten steht. In der Publikation werden die Ergebnisse von 2010 dargestellt. In den vergangenen Jahren erfolgte mit den Ergebnissen eine kontinuierliche Weiterentwicklung durch regelmäßige Qualitätszirkel, Fallbesprechungen, Aktualisierung der Schmerzschemata und Weiterbildungen zum Thema Schmerz. Eine neuerliche Evaluierung erfolgt derzeit, die Ergebnisse werden 2015 vorliegen.

\section{Take Home Message}

> Schmerzen stellen ein sehr häufiges und klinisch immens relevantes Problem bei stationär behandelten neurologischen Patienten dar, daher sollten multiprofessionelle Schmerzmanagementkonzepte in die klinische Routine eingeführt werden.

- Schmerzen bei degenerativen Erkrankungen der Wirbelsäule ohne Wurzelkompression sowie hohes Lebensalter, Einschränkungen von Mobilität und Kommunikationsfähigkeit und Schmerzen bei Mobilisation bedürfen besonderer Aufmerksamkeit in der Schmerztherapie neurologischer Patienten.

- Zur Prävention nächtlicher Schmerzspitzen sollten am Abend Analgetika mit ausreichend langer Halbwertszeit als Fixmedikation verabreicht und ein Algorithmus für eine zusätzliche Bedarfsmedikation festgelegt werden.

\section{Ergänzendes Material}

Die Therapieschemata finden Sie unter http://dx.doi.org/10.1055/ s-0034-1399111

\section{Institute}

1 Universitätsklinik für Psychiatrie, Medizinische Universität Graz, Austria

2 Universitätsklinik für Neurologie, Medizinische Universität Graz, Austria

3 Universitätsklinik für Anästhesiologie und Intensivmedizin, Medizinische Universität Graz, Austria

4 Abteilung für Schmerztherapie, Berufsgenossenschaftliches Universitätsklinikum Bergmannsheil GmbH, Bochum

Institut für Pflegewissenschaft und Praxis, Paracelsus Medizinische Privatuniversität, Salzburg, Austria

6 Stabsstelle Qualitätsmanagement-Risikomanagement, LKH-Universitätsklinikum Graz, Austria

Interessenkonflikt: Frau Mag. Christine Foussek meldet die Erstattung von Reise/Übernachtungskosten sowie eines Honorars für einen Vortrag bei einer Fortbildungsveranstaltung, bei der ein Bezug zum Thema bestand durch die Firma Mundipharma. Herr Univ.-Prof. Dr. Christoph Maier meldet finanzielle Unterstützung durch die Firmen Pfizer und Mundipharma für Beratertätigkeiten. Zusätzlich bestünde eine Unterstützung für wissenschaftliche Tätigkeiten durch den Forschungsverbund Neuropathischer Schmerz (Bundesministerium für Bildung und Forschung DLR); EU-Projekt Europain: „Understanding chronic pain and improving its treatment".

Herr Univ.-Prof. Dr. Andreas Sandner-Kiesling meldet finanzielle Unterstützung für Vortrags- und Beratertätigkeit durch die Firmen Fresenius, Mundipharma, TEVA-Ratiopharm und Grunenthal.

\section{Literatur}

1 Gerbershagen K, Gerbershagen HU, Lindena GU et al. Prevalence and impact of pain in neurological inpatients of a German teaching hospital. Clini Neurol Neurosurg 2008; 110: 710-717

2 Williams $L S$, Jones $W$, Shen J et al. Outcomes of newly referred neurology outpatients with depression and pain. Neurology 2004; 63: 674-677

3 Gerbershagen $K$, Limmroth $V$. Aspects of pain therapy: a survey of neurologic care in Germany. Der Nervenarzt 2007; 78: 923 -930

4 Maier C, Nestler N, Richter $H$ et al. The quality of pain management in German hospitals. Deutsch Arztebl Int 2010; 107: 607-614

5 Lagger V, Mahrer IR, Imhof L. Pain management in patients with cognitive impairment: a research implementation project; 2008; Pflege 21: $149-156$

6 English C, McLennan H, Thoirs $K$ et al. Reviews: Loss of skeletal muscle mass after stroke: a systematic review. Int J Stroke 2010; 5: 395 -402

7 Peterson M, Gordon P, Hurvitz E. Chronic disease risk among adults with cerebral palsy: the role of premature sarcopoenia, obesity and sedentary behaviour. Obes Rev 2013; 14: 171 - 182

8 Langhorne P, Bernhardt J, Kwakkel G. Stroke rehabilitation. The Lancet 2011; 377: $1693-1702$

9 Donovan M, Dillon P, McGuire L. Incidence and characteristics of pain in a sample of medical-surgical inpatients. Pain 1987; 30: 69-78

10 Visentin $M$, Zanolin E, Trentin $L$ et al. Prevalence and treatment of pain in adults admitted to Italian hospitals. Eur J Pain 2005; 9: 61 - 67

11 Logan SA, MacMahon E. Viral meningitis. BMJ 2008; 336: 36-40

12 van de Beek D, de Gans J, Spanjaard L et al. Clinical features and prognostic factors in adults with bacterial meningitis. N Engl J Med 2004; 351: $1849-1859$

13 Vallerand $A$. The use of long-acting opioids in chronic pain management. Nurs Clin North Am 2003; 38: 435 - 445

14 Graziotti P, Goucke R. The use of oral opioids in patients with chronic nonmalignant pain: Management strategies. Med J Austr 1997; 167: $30-34$

15 McLiesh P, Mungall D, Wiechula R. Are we providing the best possible pain management for our elderly patients in the acute-care setting? International Journal of Evidence-Based Healthcare 2009; 7: 173 - 180

16 Zedlitz AM, Visser-Meily AJ, Schepers VP et al. Patients with severe poststroke fatigue show a psychosocial profile comparable to patients with other chronic disease: implications for diagnosis and treatment. ISRN Neurology 2011; 2011: 627081

17 Thorpy MJ. Sleep disorders in Parkinson's disease. Clinical Cornerstone 2004; 6: $7-15$ 
18 Fleming WE, Pollak $C P$. Sleep disorders in multiple sclerosis. Semin Neurol 2005: 64-68

19 Hess JH. Postdural puncture headache: A literature review. AANA journal 1991; 59: 549-555

20 Morewood GH. A rational approach to the cause, prevention and treatment of postdural puncture headache. CMAJ: Canadian Medical Association Journal 1993; 149: 1087-1093

21 Hendrick P, Te Wake A, Tikkisetty A et al. The effectiveness of walking as an intervention for low back pain: a systematic review. Eur Spine J 2010; 19: 1613 - 1620

22 Kwon B, Roffey D, Bishop P et al. Systematic review: occupational physical activity and low back pain. Occup Med 2011; 61: 541 - 548

23 Roffey DM, Wai EK, Bishop P. Causal assessment of occupational standing or walking and low back pain: results of a systematic review. Spine J 2010; 10: 262 - 272

24 Horn AI, Fontes SV, Carvalho SMRd et al. Kinesiotherapy prevents shoulder pain in hemiplegic/paretic patients on sub-acute stage poststroke. Arq Neuropsiquiatria 2003; 61: 768 - 771
25 Chapman S. Managing pain in the older person. Nurs Stand 2010; 25: 35-39

26 Arnstein P. Balancing analgesic efficacy with safety concerns in the older patient. Pain Manag Nurs 2010; 11: $11-22$

27 Fine $P G$. Treatment guidelines for the pharmacological management of pain in older persons. Pain Med 2012; 13: 57-66

28 Norrbrink C, Löfgren M, Hunter JP et al. Patients' Perspectives on Pain. Top Spinal Cord Inj Rehabil 2012; 18: 50 -56

29 Schofield P. Pain assessment and management in older hospitalised patients: observation shows common themes relating to nurse-patient communication, pain management strategies, organisational aspects of care and the complex nature of pain. Evid Based Nurs 2013

30 Davies-Osterkamp S, Salm A. Ansätze zur Erfassung psychischer Adaptationsprozesse in medizinischen Belastungssituationen. Med Psychol 1980; 6: $66-80$ 